\title{
CORRIGENDUM
}

\section{ON COMPONENTS OF STABLE AUSLANDER-REITEN QUIVERS THAT CONTAIN HELLER LATTICES: THE CASE OF TRUNCATED POLYNOMIAL RINGS - CORRIGENDUM}

\author{
SUSUMU ARIKI, RYOICHI KASE AND KENGO MIYAMOTO
}

Published online by Cambridge University Press 05 December 2016, doi:10.1017/nmj.2016.53

The original publication of $[\mathrm{AKM}]$ contained several errors that the authors wish to correct in the following.

Throughout, $\mathcal{O}$ will be a complete discrete valuation ring, and $A$ will be a symmetric $\mathcal{O}$-order (refer to [CR, Definition 23.1] for the definition of an $\mathcal{O}$-order). We write $l a t t$ - $A$ for the category of $A$-lattices and the stable Auslander-Reiten quiver of $A$ means that for latt- $A$.

In $[\mathrm{AKM}]$, we introduced the stable Auslander-Reiten quiver of $A$ and determined stable components of $A$ that contain Heller lattices for the case $A=\mathcal{O}[X] /\left(X^{n}\right)$. The final result Theorem 3.1 is correct, but preparatory results Lemma 1.23 and Theorem 1.27 need to be stated more precisely. We correct those parts in this corrigendum. We also correct the careless statement of Lemma 1.21(3) in the original paper.

\section{§1.1. On [AKM, Definition 1.20 and Lemma 1.21].}

Let $(\Delta, v)$ be a valued quiver. We denote the map $v: \Delta_{1} \rightarrow \mathbb{Z}_{>0} \times \mathbb{Z}_{>0}$ by $v(x \rightarrow y)=\left(d_{x y}, d_{x y}^{\prime}\right)$. In this paper, we assume that $\Delta$ does not have loops, two cycles and multiple arrows. Therefore, underlying undirected graph of the quiver is a simple graph, and each edge is given an orientation. In other words, if two vertices $x$ and $y$ are connected by an arrow then $x \neq y$ and either $x \rightarrow y$ or $y \rightarrow x$ holds exclusively. In this setting, the valued quiver $(\Delta, v)$ gives rise to the Cartan matrix $C=\left(c_{x y}\right)_{x, y \in \Delta_{0}}$ on $\Delta_{0}$ below, since

2010 Mathematics subject classification. 16G70, 16G30.

(C) 2019 Foundation Nagoya Mathematical Journal 
$c_{x y} \neq 0$ if and only if $c_{y x} \neq 0$ holds.

$$
c_{x y}:= \begin{cases}2 & \text { if } x=y \\ -d_{x y} & \text { if } x \in y^{-} \\ -d_{y x}^{\prime} & \text { if } x \in y^{+} \\ 0 & \text { otherwise }\end{cases}
$$

where $y^{+}=\left\{x \in \Delta_{0} \mid y \rightarrow x \in \Delta_{1}\right\}$ and $y^{-}=\left\{x \in \Delta_{0} \mid x \rightarrow y \in \Delta_{1}\right\}$. The following is a well-known statement about Cartan matrices, which we may find in [B, Theorem 4.5.8].

LEMmA 1.21. Let $(\Delta, v)$ be a connected valued quiver as above and $\bar{\Delta}$ the underlying graph of $\Delta$.

(1) Assume that $(\Delta, v)$ admits a function $f: \Delta_{0} \rightarrow \mathbb{Q}_{>0}$ which satisfies

$$
2 f(y) \geqslant \sum_{x \in y^{-}} f(x) d_{x y}+\sum_{x \in y^{+}} f(x) d_{y x}^{\prime}, \quad \text { for all } y \in \Delta_{0}
$$

or, equivalently, $\sum_{x \in \Delta_{0}} f(x) c_{x y} \geqslant 0$, for all $y \in \Delta_{0}$. Then the following hold.

(i) If $\Delta$ has a finite number of vertices, then $\bar{\Delta}$ is one of the finite or affine Dynkin diagrams.

(ii) If $\Delta$ has infinite number of vertices, then $\bar{\Delta}$ is one of the infinite Dynkin diagrams $A_{\infty}, B_{\infty}, C_{\infty}, D_{\infty}$ or $A_{\infty}^{\infty}$.

(2) If the inequality is strict for a vertex of $\Delta$, then $\bar{\Delta}$ is either a finite Dynkin diagram or $A_{\infty}$.

(3) If $f$ is unbounded, then $\bar{\Delta}$ is $A_{\infty}$.

\section{§1.2. On [AKM, Lemma 1.23]}

Lemma 1.23 states that there are no loops in periodic stable components with infinitely many vertices of $A$. However, the statement is false in the general setting assumed in Lemma 1.23. We correct the statement of [AKM, Lemma 1.23] and give a proof by modifying the original proof.

Lemma 1.23. Let $C$ be a component of the stable Auslander-Reiten quiver of $A$. Assume that $C$ satisfies the following conditions:

(i) There exists a $\tau$-periodic indecomposable A-lattice in $C$.

(ii) $C$ has infinitely many vertices. 
Then $C \backslash\{$ loops $\}$ is of the form $\mathbb{Z} A_{\infty} /\langle\tau\rangle$ if $C$ has a loop. In this case, there exists exactly one loop and it appears at the endpoint of $C$ such that the valuation of the loop is trivial.

Proof. First, we show that if $X \in C$ has a loop, then $X \simeq \tau X$. Suppose that $X \in C$ has a loop and $X \not \tau X$. Then the almost split sequence ending at $X$ is of the form

$$
0 \rightarrow \tau X \rightarrow X^{\oplus l_{1}} \oplus E_{X} \oplus \tau X^{\oplus l_{2}} \rightarrow X \rightarrow 0,
$$

where $E_{X} \in$ latt- $A$ and $l_{1}, l_{2} \geqslant 1$. Therefore,

$$
\operatorname{rank}(X)+\operatorname{rank}(\tau X)=l_{1} \operatorname{rank}(X)+l_{2} \operatorname{rank}(\tau X)+\operatorname{rank}\left(E_{X}\right),
$$

implies $\operatorname{rank}\left(E_{X}\right)=0$ and $l_{1}=l_{2}=1$. However, it follows from [M, Theorem 1] that the almost split sequence ending at $X$ splits, a contradiction. Therefore, if $X$ has a loop, then $X$ and $\tau X$ are isomorphic.

As in the proof of [AKM, Lemma 1.23], which was taken from [HPR], we choose $n_{X} \geqslant 1$, for each $X \in C$, such that $\tau^{n_{X}} X \simeq X$, and define $f: C_{0} \rightarrow$ $\mathbb{Q}_{>0}$ by

$$
f(X)=\frac{1}{n_{X}} \sum_{i=0}^{n_{X}-1} \operatorname{rank}\left(\tau^{i} X\right) .
$$

Note that $f(X)=f(\tau X)$ holds, for all $X \in C$. Set $\widetilde{C}:=C \backslash\{$ loops $\}$. By the Riedtmann structure theorem [B, Theorem 4.15.6], there exist a directed tree $T$ and an admissible group $G$ such that $\widetilde{C}=\mathbb{Z} T / G$. We denote the $G$-orbit of $(t, r)$, for a vertex $t$ of $T$ and $r \in \mathbb{Z}$, by $\overline{(t, r)}$. Let $X_{t}=\overline{(t, 0)}$. Then, we define a function $\tilde{f}: T_{0} \rightarrow \mathbb{Q}>0$ by $\tilde{f}(t)=f\left(X_{t}\right)$. By considering the almost split sequence

$$
0 \rightarrow \tau X_{t} \rightarrow\left(\bigoplus_{s \in t^{-}} X_{s}^{d_{s t}}\right) \oplus\left(\bigoplus_{s \in t^{+}} \tau X_{s}^{d_{t s}^{\prime}}\right) \oplus X_{t}^{l} \oplus \tau X_{t}^{l^{\prime}} \rightarrow X_{t} \rightarrow 0
$$

where $l, l^{\prime} \geqslant 0$, we have

$$
2 \widetilde{f}(t) \geqslant \sum_{s \in t^{-}} \widetilde{f}(s) d_{s t}+\sum_{s \in t^{+}} \widetilde{f}(s) d_{t s}^{\prime}
$$

Now, suppose that $X=\overline{(t, r)} \in C$ has a loop. It implies that the above inequality is strict for $t$. Thus, by the assumption (ii) and Lemma 1.21(2), 
the underlying tree $\bar{T}$ is $A_{\infty}$ so that $\widetilde{C}=\mathbb{Z} A_{\infty} /\langle\tau\rangle$. We may assume without loss of generality that $T$ has a linear orientation, that is, $T$ does not have a sink. Thus, we may take a path in $\widetilde{C}$

$$
X_{1} \rightarrow X_{2} \rightarrow \cdots \rightarrow X_{r} \rightarrow \cdots
$$

We assume that $X_{r}$ has a loop. If $r>1$ then the almost split sequence starting at $X_{r}$ is

$$
0 \longrightarrow X_{r} \longrightarrow X_{r}^{\oplus l} \oplus X_{r+1} \oplus X_{r-1} \oplus P \longrightarrow X_{r} \longrightarrow 0
$$

where $l \geqslant 1$ and $P$ is a projective $A$-lattice. Since $f\left(X_{t}\right) \geqslant 1$ for all $t \geqslant 1$, we have

$$
f\left(X_{r}\right) \geqslant(2-l) f\left(X_{r}\right) \geqslant f\left(X_{r+1}\right)+f\left(X_{r-1}\right) \geqslant f\left(X_{r+1}\right)+1 .
$$

We show that $f\left(X_{m}\right) \geqslant f\left(X_{m+1}\right)+1$ for $m \geqslant r$. Suppose that $f\left(X_{m-1}\right) \geqslant$ $f\left(X_{m}\right)+1$ holds. The same argument as above shows $2 f\left(X_{m}\right) \geqslant f\left(X_{m-1}\right)+$ $f\left(X_{m+1}\right)$, and the induction hypothesis implies

$$
2 f\left(X_{m}\right) \geqslant f\left(X_{m-1}\right)+f\left(X_{m+1}\right) \geqslant f\left(X_{m}\right)+f\left(X_{m+1}\right)+1,
$$

so that $f\left(X_{m}\right) \geqslant f\left(X_{m+1}\right)+1$ follows. Thus, there exists a positive integer $t$ such that $f\left(X_{t}\right)<0$, which contradicts with $f\left(X_{t}\right) \geqslant 1$. We conclude that $r=1$. Then, $l=1$ by $2 \operatorname{rank} X_{1}>l \operatorname{rank} X_{1}$. We have proved that the loop is unique and it appears at the endpoint of the homogeneous tube such that the valuation is $(1,1)$.

\section{§1.3. On [AKM, Theorem 1.27]}

In the proof of Theorem 1.27, we used Lemma 1.23 to ensure that $\tau$ periodic stable components of $A$ with infinitely many vertices do not admit a loop. However, there is a possibility that a loop appears at the endpoint of a homogeneous tube in the general setting of Theorem 1.27. Hence we correct the statement of [AKM, Theorem 1.27] as follows.

THEOREM 1.27. Let $A$ be a symmetric $\mathcal{O}$-order over a complete discrete valuation ring $\mathcal{O}$, and let $C$ be a component of the stable Auslander-Reiten quiver of $A$. Suppose that:

(i) there exists a $\tau$-periodic indecomposable A-lattice in $C$;

(ii) the stable Auslander-Reiten quiver of A has infinitely many vertices. 
Then, the number of vertices of $C$ is infinite, and either:

(a) $C$ is a valued stable translation quiver; or

(b) a loop with trivial valuation appears at the endpoint of $C$ and if we delete the loop, then $C$ becomes a homogeneous tube $\mathbb{Z} A_{\infty} /\langle\tau\rangle$.

\section{§1.4. The case of $A=\mathcal{O}[X] /\left(X^{n}\right)$}

Now we apply Theorem 1.27 to a component of the stable AuslanderReiten quiver of $A=\mathcal{O}[X] /\left(X^{n}\right)$ that contains a Heller lattice. If there exists a loop in the component, Proposition 2.4 implies that the endpoint must be a Heller lattice. However, Heller lattices have no loops. Thus any component of the stable Auslander-Reiten quiver of $A$ that contains a Heller lattice is a valued stable translation quiver, so that the proof of [AKM, Theorem 3.1] works without change.

\section{REFERENCES}

[AKM] S. Ariki, R. Kase and K. Miyamoto, On components of stable Auslander-Reiten quivers that contain Heller lattices: the case of truncated polynomial rings, Nagoya Math. J. 228 (2017), 72-113.

[B] D. Benson, "Representations and cohomology. I", in Basic Representation Theory of Finite Groups and Associative Algebras, Cambridge Studies in Advanced Mathematics 30, Cambridge University Press, Cambridge, 1991.

[CR] C. W. Curtis and I. Reiner, "Methods of representation theory", in With Applications to Finite Groups and Orders, Pure and Applied Mathematics I, A Wiley-Interscience Publication. John Wiley \& Sons, Inc., New York, 1981.

[HPR] D. Happel, U. Preiser and C. M. Ringel, "Vinberg's characterization of Dynkin diagrams using subadditive functions with application to DTr-periodic modules. Representation theory, I", in (Proc. Second Internat. Conf., Carleton Univ., Ottawa, Ont., 1979), Lecture Notes in Mathematica 832, Springer, Berlin, 1980, 280-294.

[M] T. Miyata, Note on direct summands of modules, J. Math. Kyoto Univ. 7 (1967), $65-69$.

Susumu Ariki

Department of Pure and Applied Mathematics

Graduate School of Information Science and Technology

Osaka University

1-5 Yamadaoka

Suita

Osaka 565-0871

Japan

ariki@ist.osaka-u.ac.jp 
Ryoichi Kase

Faculty of Informatics

Okayama University of Science

1-1 Ridaicho

Kita-ku

Okayama

Okayama 700-0005

Japan

r-kase@mis.ous.ac.jp

Kengo Miyamoto

Department of Pure and Applied Mathematics

Graduate School of Information Science and Technology

Osaka University

1-5 Yamadaoka

Suita

Osaka 565-0871

Japan

k-miyamoto@ist.osaka-u.ac.jp 